\title{
Using Semantic Authoring for Blissymbols Communication Boards
}

\author{
Yael Netzer \\ Dept. of Computer Science \\ Ben Gurion University \\ Beer Sheva, Israel \\ yaelnecs.bgu.ac.il
}

\author{
Michael Elhadad \\ Dept. of Computer Science \\ Ben Gurion University \\ Beer Sheva, Israel \\ elhadadecs.bgu.ac.il
}

\begin{abstract}
Natural language generation (NLG) refers to the process of producing text in a spoken language, starting from an internal knowledge representation structure. Augmentative and Alternative Communication (AAC) deals with the development of devices and tools to enable basic conversation for language-impaired people. We present an applied prototype of an AAC-NLG system generating written output in English and Hebrew from a sequence of Bliss symbols. The system does not "translate" the symbols sequence, but instead, it dynamically changes the communication board as the choice of symbols proceeds according to the syntactic and semantic content of selected symbols, generating utterances in natural language through a process of semantic authoring.
\end{abstract}

\section{Introduction}

People who suffer from severe language impairments lack the ability to express themselves through natural usage of language and cannot achieve various forms of communication. The field of Augmentative and Alternative Communication (AAC) is concerned with methods that can be added to the natural communication. In the most common form, iconic symbols are presented on a display (or a communication board). Communication is conducted by the sequential selection of symbols on the display (with vocal output when available), which are then interpreted by the partner in the interaction.

AAC devices are characterized by three aspects: (i) Selection method i.e., the physical choice of symbols on the communication board; (ii) input language and (iii) output medium. In a computerized system, as (McCoy and Hershberger, 1999) mention, a processing method aspect is added to this list. This method refers to the process which creates the output once symbols are inserted.

We specifically study the set of symbols (as an input language) called Blissymbolics (Bliss in short). Bliss is a graphic meaning-referenced language, created by Charles Bliss to be used as a written universal language (Bliss, 1965); since 1971, Blissymbols are used for communication with severely languageimpaired children. Bliss is designed to be a writtenonly language, with non-arbitrary symbols. Symbols are constructed from a composition of atomic icons. Because words are structured from semantic components, the graphic representation by itself provides information on words' connectivity ${ }^{1}$.

In the last decade, several systems that integrate NLG techniques for AAC systems have been developed ((McCoy, 1997), (Vaillant, 1997) for example). These systems share a common architecture: a telegraphic input sequence (words or symbols) is first parsed, and then a grammatical sentence that represents the message is generated.

This paper presents an NLG-AAC system that generates messages through a controlled process of authoring, where each step in the selection of symbols is controlled by the input specification defined

\footnotetext{
${ }^{1}$ See http://www.bci.org for reference on the language
} 
for the linguistic realizer.

\section{Generating Messages via Translation}

A major difficulty when parsing a telegraphic sequence of words or symbols, is that many of the hints that are used to capture the structure of the text and, accordingly, the meaning of the utterance, are missing. Moreover, as an AAC device is usually used for real-time conversation, the interpretation of utterances relies heavily on pragmatics - time of mentioned events, reference to the immediate environment.

Previous works dealing with translating telegraphic text, such as (Grishman and Sterling, 1989), (Lee et al., 1997) requires to identify dependency relations among the tokens of the telegraphic input. Rich lexical knowledge is needed to identify possible dependencies in a given utterance, i.e., to find the predicate and to apply constraints, such as selectional restrictions to recognize its arguments.

Similar methods were used for AAC applications, COMPANSION (McCoy, 1997) for example - where the telegraphic text is expanded to full sentences, using a word order parser, and a semantic parser to build the case frame structure of the verb in the utterance, filling the slots with the rest of the content words given. The system uses the semantic representation to re-generate fluent text, relying on lexical resources and NLG techniques.

The main questions at stake in this approach are how good can a semantic parser be, in order to reconstruct the full structure of the sentence from telegraphic input and are pragmatic gaps in the given telegraphic utterances recoverable in general.

\section{Generating Messages via Semantic Authoring}

Our approach differs from previous NLG-AAC systems in that, with the model of semantic authoring (Biller et al., 2005), we intervene during the process of composing the input sequence, and thus can provide early feedback (in the form of display composition and partial text feedback), while preventing the need for parsing a telegraphic sequence.

Semantic parsing is avoided by constructing a semantic structure explicitly while the user inputs the sequence incrementally. It combines three aspects into an integrated approach for the design of an AAC system:

- Semantic authoring drives a natural language realization system and provides rich semantic input.

- A display is updated on the fly as the authoring system requires the user to select options.

- Ready-made inputs, corresponding to predefined pragmatic contexts are made available to the user as semantic templates.

In this method, each step of input insertion is controlled by a set of constraints and rules, which are drawn from an ontology. The system offers, at each step, only possible complements to a small set of concepts. For example, if the previous symbol denotes a verb which requires an instrumental theme, only symbols that can function as instruments are presented on the current display. Other symbols are accessible through navigation operations, which are interpreted in the context of the current partial semantic specification. The general context of each utterance or conversation can be determined by the user, therefore narrowing the number of symbols displayed in the board.

The underlying process of message generation is based on layered lexical knowledge bases (LKB) and an ontology. The ontology serves as a basis for the semantic authoring process; it includes a hierarchy of concepts and relations, and the information it encodes interacts with the conceptual graphs processing performed as part of content determination and lexical choice. The ontology was acquired with a semi-automatic tool, which relies on WordNet (Miller, 1995) and VerbNet (Kipper et al., 2000).

We designed and implemented the Bliss lexicon for both Hebrew and English. The lexicon can be used either as a stand-alone lexicon or as part of an application through an API. The design of the lexicon takes advantage of the unique properties of the language. The Bliss lexicon provides the list of symbols accessible to the user, along with their graphic representation, semantic information, and the mapping of symbols to English and Hebrew words. The lexicon can be searched by keyword (learn), or by semantic/graphic component: searching all words in the lexicon that contain both food and meat returns the symbols hamburger, hot-dog, meatball etc. (see 
Fig. 1). The lexicon currently includes 2,200 entries.

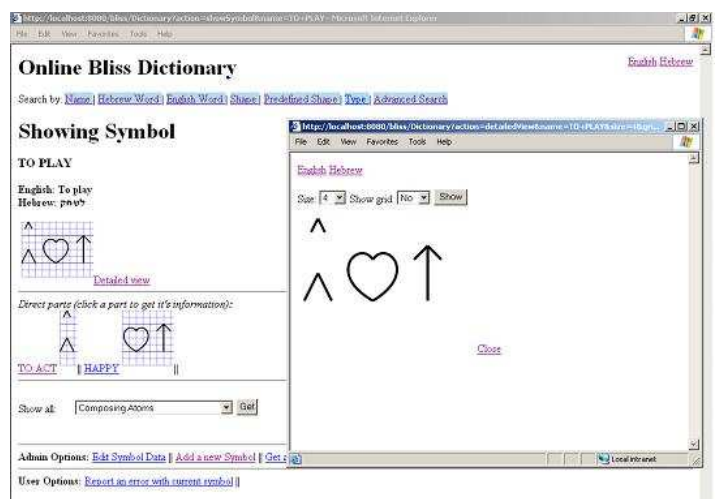

Figure 1: A snapshot of the Bliss Lexicon Web Application

The core of the processing machinery of the AAC message generation system is based on SAUT (Biller et al., 2005) - an authoring system for logical forms encoded as conceptual graphs (CG). The system belongs to the family of WYSIWYM (What You See Is What You Mean) (Power and Scott, 1998) text generation systems: logical forms are entered interactively and the corresponding linguistic realization of the expressions is generated in several languages. The system maintains a model of the discourse context corresponding to the authored documents to enable reference planning in the generation process.

Generating language from pictorial inputs, and specifically from Bliss symbols using semantic authoring in the WYSIWYM approach is not only a pictorial application of the textual version, but it also addresses specific needs of augmentative communication.

As was mentioned above, generating text from a telegraphic message for AAC usage must take the context of the conversation into account. We address this problem in two manners: (1) adding pre-defined inputs into the system (yet allowing accurate text generation that considers syntactic variations), and (2) enabling the assignment of default values to each conversation (such as participants, tense, mood). We also take advantage of the unique properties of the Bliss symbols; the set of symbols that are offered in each display can be filtered using their semantic/graphical connectivity; the reduction of the number of possible choices that are to be made by the user in each step of the message generation affects the cognitive load and can affect the rate of communication.

\section{Evaluation}

We evaluate our system as an AAC application for message generation from communication boards. From an NLG evaluation perspective, this corresponds to an intrinsic evaluation, i.e. judging quality criteria of the generated text and its adequacy relative to the input (Bangalore et al., 1998). Since the prototype of our system is not yet adjusted to interact with alternative pointing devices, we could not test it on actual Bliss users, and could not perform a full extrinsic (task-based) evaluation.

However, as argued in (Higginbotham, 1995), evaluations of AAC systems with nondisabled subjects, when appropriately used, is easier to perform, and in some cases provide superior results. Higginbotham's claims rely on the observation that the methods of message production are not unique to AAC users and analogous communication situations exist both for disabled and nondisabled users. Nondisabled subjects can contribute to the understanding of the cognitive processes underlying the acquisition of symbol and device performance competencies. We believe that the evaluation of efficiency for non-AAC users should be served as baseline.

The approach we offer for message generation requires users to plan their sentences abstractly. (McCoy and Hershberger, 1999) points that novel systems may be found to slow communication but to increase literacy skills. We therefore tested both speed of message generation and semantic coverage (the capability to generate a given message correctly).

The usage of semantic authoring was evaluated on nondisabled subjects through a user study of 10 subjects. This provides a reliable approximation of the learning curve and usability of the system in general (Biller et al., 2005).

In order to evaluate the keystroke savings of the system we have collected a set of 19 sentences written in Bliss and their full English correspondents. We compared the number of the words in the English sentences with the number of choices needed to generate the sentence with our system. The total number of choice steps is 133 , while the total num- 
ber of words in the sentences is 122 . This simple ratio shows no improvement of keystrokes saving using our system. Savings, therefore, must be calculated in terms of narrowing the choice possibilities in each step of the process.

However, counting the number of words does not include morphology which in Bliss symbols requires additional choices. We have counted the words in the sentences considering morphology markers of inflections as additional words, all summing to 138, as was suggested in (McCoy and Hershberger, 1999).

Assuming a display with 50 symbols (and additional keys for functions) - a vocabulary of requires 50 different screens. Assuming symbols are organized by frequencies (first screens present the most frequently used words) or by semantic domain.

The overall number of selections is reduced using our communication board since the selectional restrictions narrow the number of possible choices that can be made at each step. The extent to which selection time can be reduced at each step depends on the application domain and the ontology structure. We cannot evaluate it in general, but expect that a wellstructured ontology could support efficient selection mechanisms, by grouping semantically related symbols in dedicated displays.

In addition, the semantic authoring approach can generate fluent output in other languages (English and Hebrew, beyond the Bliss sequence - without requiring noisy translation). We also hypothesize that ontologically motivated grouping of symbols could speed up each selection step - but this claim must be assessed empirically in a task-based extrinsic evaluation, which remains to be done in the future.

We are now building the environment for AAC users with cooperation with ISAAC-ISRAEL ${ }^{2}$, in order to make the system fully accessible and to be tested by AAC-users. However, this work is still in progress. Once this will be achieved, full evaluation of the system will be plausible.

\section{Conclusions and Future Work}

This work offers a new approach for message generation in the context of AAC displays using seman-

\footnotetext{
${ }^{2}$ Israeli chapter of the International Society for Augmentative and Alternative Communication
}

tic authoring and preventing the need to parse and re-generate. We have designed and implemented a Bliss lexicon for both Hebrew and English, which can either be used a stand-alone lexicon for reference usage or as a part of an application.

Future work includes an implementation of a system with full access for alternative devices, expansion of the underlying lexicon for Hebrew generation, and adding voice output.

\section{References}

Srinivas Bangalore, Anoop Sarkar, Christy Doran, and BethAnn Hockey. 1998. Grammar and parser evaluation in the XTAG project. In Proc. of Workshop on Evaluation of Parsing Systems, Granada, Spain, May.

Ofer Biller, Michael Elhadad, and Yael Netzer. 2005. Interactive authoring of logical forms for multilingual generation. In Proc. of the 10th workshop of ENLG, Aberdeen, Scotland.

Charles K. Bliss. 1965. Semantography (Blissymbolics). Semantography Press, Sidney.

Ralph Grishman and John Sterling. 1989. Analyzing telegraphic messages. In Proc. of DARPA Speech and Natural Language Workshop, pages 204-208, Philadelphia, February.

D. Jeffery Higginbotham. 1995. Use of nondisabled subjects in AAC research: Confessions of a research infidel. AAC Augmentative and Alternative Communication, 11, March. AAC Research forum.

K. Kipper, H. Trang Dang, and M. Palmer. 2000. Class-based construction of a verb lexicon. In Proceeding of AAAI-2000.

Young-Suk Lee, Clifford Weinstein, Stephanie Seneff, and Dinesh Tummala. 1997. Ambiguity resolution for machine translation of telegraphic messages. In Proc. of the 8th conference on EACL, pages 120-127.

Kathleen F. McCoy and Dave Hershberger. 1999. The role of evaluation in bringing NLP to AAC: A case to consider. In Filip T. Loncke, John Clibbens, Helen H. Arvidson, and Lyle L. Lloyd, editors, AAC: New Directions in Research and Practice, pages 105-122. Whurr Publishers, London.

Kathleen F. McCoy. 1997. Simple NLP techiques for expanding telegraphic sentences. In Proc. of workshop on NLP for Communication Aids, Madrid, July. ACL/EACL.

George A. Miller. 1995. WordNET: a lexical database for English. Commun. ACM, 38(11):39-41.

Roger Power and Donia Scott. 1998. Multilingual authoring using feedback texts. In Proc. of COLING-ACL 98, Montreal, Canada.

Pascal Vaillant. 1997. A semantic-based communication system for dysphasic subjects. In Proc. of the 6th conference on AI in Medicine Europe (AIME'97), Grenoble, France, March. 